Pacific Journal of Mathematics

DECOMPOSITIONS OF $E^{3}$ INTO POINTS AND COUNTABLY RICHARD SNAP 


\title{
DECOMPOSITIONS OF $E^{3}$ INTO POINTS AND COUNTABLY MANY FLEXIBLE DENDRITES
}

\author{
RICHARD A. SNAY
}

\begin{abstract}
Let $G$ be an upper semicontinuous decomposition of $E^{3}$ whose only nondegenerate elements are countably many dendrites. It has been asked by Armentrout whether it is sufficient that each dendrite be tame in $E^{3}$ in order that the decomposition space $E^{3} \mid G$ be homeomorphic to $E^{3}$. In Theorem 3 the sufficiency of the tameness condition is shown as well as the sufficiency of the weaker condition that each dendrite be flexible in $E^{3}$. Theorem 2 states that if $A$ and $B$ are flexible dendrites in $E^{3}$ whose intersection is a point, then $A \cup B$ is a flexible dendrite. This result is used to construct flexible dendrites in $E^{3}$ which are not tame.
\end{abstract}

An upper semicontinuous decomposition $G$ of a topological space $X$ is a collection of disjoint subsets of $X$ such that $X$ is the union of elements of $G$ and such that for every $g \in G$ and for every open set $U$ in $X$ containing $g$, there is an open set $V$ in $X$ such that $g \subset V \subset U$ and $V$ is the union of elements of $G$. The decomposition space of $X$ associated with $G$, denoted $X / G$, is the set $G$ with the topology defined by the condition that a subset $W$ of $G$ is open in $X / G$ if and only if the union of the elements of $W$ is open in $X$. A dendrite is a locally connected continuum which contains no simple closed curve. A tree is a finite 1-dimensional simplicial complex whose geometric realization is a dendrite. If $M$ is an $n$-manifold with or without boundary, Int $M$ denotes the set consisting of all points of $M$ which have a neighborhood homeomorphic to $E^{n}$, and $\mathrm{Bd} M$ denotes $M$ - Int $M$. If $U$ is a subset of the space $X$, then $\mathrm{Cl} U$ denotes the closure of $U$ in $X$.

Definition. A dendrite $K$ in $E^{3}$ is tame if there is a homeomorphism $h$ of $E^{3}$ onto itself such that $h(K)$ is a subset of the $x y$ plane.

Definition. A dendrite $K$ in $E^{3}$ is flexible if given two subcontinua $K_{1}$ and $K_{2}$ such that $K=K_{1} \cup K_{2}$ and given two open sets $U_{1}$ and $U_{2}$ in $E^{3}$ such that $K_{i} \subset U_{i}(i=1,2)$, then there is a homeomorphism $f$ of $E^{3}$ onto itself such that $f(K) \subset U_{2}$ and $f$ is the identity on $E^{3}-U_{1}$.

REMARK. Observe that if $K$ is a dendrite in $E^{3}$ and if $h$ is a 
homeomorphism of $E^{3}$ onto itself, then $K$ is flexible if and only if $h(K)$ is flexible.

Lemma 1. Let $D$ be a disk contained in the xy-plane $P$ of $E^{3}$. If $U$ is an open set in $E^{3}$ containing $D$ and if $g$ is a homeomorphism of $D$ onto itself which is the identity on $B d D$, then there is a homeomorphism $f$ of $E^{3}$ onto itself such that $f$ equals $g$ on $D$ and $f$ is the identity on $\left(E^{3}-U\right) \cup(P-D)$.

Proof. Let $h$ be a homeomorphism of $E^{3}$ onto itself such that $h(D)=\left\{(x, y, z) \in E^{3}: x^{2}+y^{2} \leqq 1\right.$ and $\left.z=0\right\}$. Since $h(U)$ contains $h(D)$, there is a positive number $\varepsilon$ such that the suspension $S$ of $h(D)$ with respect to the points $(0,0, \varepsilon)$ and $(0,0,-\varepsilon)$ is contained in $h(U)$. Let $k$ be the homeomorphism of $E^{3}$ onto itself which equals the suspension of $h \cdot g \cdot h^{-1} \mid h(D)$ on $S$ and which equals the identity elsewhere. Then $f$ equal to $h^{-1} \circ k \circ h$ is the required homeomorphism.

Theorem 1. If $K$ is a tame dendrite in $E^{3}$, then $K$ is flexible.

Proof. Since flexibility is invariant under homeomorphisms of $E^{3}$ onto itself, we may assume that $K$ is a subset of the $x y$-plane $P$ in $E^{3}$. Let $K_{1}$ and $K_{2}$ be subcontinua of $K$ such that $K=K_{1} \cup K_{2}$ and let $U_{1}$ and $U_{2}$ be open sets in $E^{3}$ such that $K_{i} \subset U_{i}(i=1,2)$. Let

$$
\varepsilon=\min \left\{\operatorname{dist}\left(K_{1}, E^{3}-U_{1}\right), \quad \operatorname{dist}\left(K_{2}, E^{3}-U_{2}\right)\right\}
$$

and let $T$ be a triangulation of $P$ of mesh less than $\varepsilon$ such that the O-skeleton on $T$ misses $K$. Since $K$ does not separate $P$, there is a polyhedral disk $D$ in $P$ such that $K \subset \operatorname{Int} D, D$ misses the 0 -skeleton of $T$, and $\mathrm{Bd} D$ is in general position with the 1-skeleton of $T$ in $P$. Hence if $s$ is a closed 2-simplex of $T$, then the components of $s \cap D$ consist of finitely many disjoint polyhedral disks. Let $\left\{D_{i}\right\}_{i=1}^{n}$ be the set of disks in $P$ such that for each $i(1 \leqq i \leqq n)$ there is a closed 2-simplex $s$ in $T$ such that $D_{i}$ is a component of $s \cap D$ and $D_{i} \cap K \neq \varnothing$. Hence $\left\{D_{i}\right\}_{i=1}^{n}$ is a set of polyhedral disks in $P$ such that:

(1) $\operatorname{diam} D_{i}<\varepsilon(1 \leqq i \leqq n)$,

(2) if $D_{i} \cap D_{j} \neq \varnothing$, then $D_{i} \cap D_{j}$ is an arc for $i \neq j$, and

(3) the nerve of $\left\{D_{i}\right\}_{i=1}^{n}$ is a tree.

By conditions (2) and (3) we have that the union of all elements of $\left\{D_{i}\right\}_{i=1}^{n}$ which meet $K_{2}$ is a disk $E$ and that the union of all elements of $\left\{D_{i}\right\}_{i=1}^{n}$ which are not subdisks of $E$ consists of disjoint disks $F_{1}, \cdots, F_{m}$ such that for each $i(1 \leqq i \leqq m) F_{i} \cap E=\operatorname{Bd} F_{i} \cap \operatorname{Bd} E$ is an arc $J_{i}$. It follows that $K \cap \mathrm{Bd} F_{i} \subset J_{i}$. By condition (1) and our choice of $\varepsilon$ we have $E \subset U_{2}$ and $F_{i} \subset U_{1}(1 \leqq i \leqq m)$. Since 
$\left(K \cap \operatorname{Bd} F_{i}\right) \subset J_{i} \subset E \subset U_{2}$, there is a homeomorphism $g_{i}$ of $F_{i}$ onto itself which is the identity on $\mathrm{Bd} F_{i}$ such that $g_{i}\left(K \cap F_{i}\right) \subset U_{2}$. The homeomorphism $g_{i}$ is obtained as follows. Choose arcs $A_{i}$ and $B_{i}$ in $F_{i}$ such that:

(a) $A_{i} \cap \mathrm{Bd} F_{i}=B_{i} \cap \mathrm{Bd} F_{i}=\mathrm{Bd} J_{i}=\mathrm{Bd} A_{i}=\mathrm{Bd} B_{i}$,

(b) the disk on $F_{i}$ bounded by $A_{i} \cup J_{i}$ contains $K \cap F_{i}$, and

(c) the disk on $F_{i}$ bounded by $B_{i} \cup J_{i}$ is contained in $U_{2}$. Now let $h_{i}$ be an embedding of $A_{i} \cup \mathrm{Bd} F_{i}$ into $F_{i}$ which is the inclusion on $\mathrm{Bd} F_{i}$ and which takes $A_{i}$ onto $B_{i}$. The homeomorphism $g_{i}$ is an extension of $h_{i}$ to all of $F_{i}$.

Now let $V_{1}, \cdots, V_{m}$ be disjoint open sets in $U_{1}$ such that $F_{i} \subset V_{i}$ $(1 \leqq i \leqq m)$. By Lemma 1 there is a homeomorphism $f_{i}$ of $E^{3}$ onto itself such that $f_{i}$ equals $g_{i}$ on $F_{i}$ and $f_{i}$ is the identity on $\left(E^{3}-V_{i}\right) \cup$ $\left(P-F_{i}\right)$. If $f$ equals $f_{m} \circ f_{m-1} \circ \cdots \circ f_{1}$, then $f(K) \subset U_{2}$ and $f$ is the identity on $E^{3}-U_{1}$. Hence $K$ is flexible.

Lemma 2. Let $K$ be a flexible dendrite in $E^{3}$. If $N, C_{1}, C_{2}, \cdots, C_{n}$ are subcontinua of $K$ and $U, V_{1}, V_{2}, \cdots, V_{n}$ are open sets in $E^{3}$ such that:

(1) $K=N \cup\left(\bigcup_{i=1}^{n} C_{i}\right)$,

(2) $N \subset U$ and $C_{i} \subset V_{i}(1 \leqq i \leqq n)$, and

(3) $V_{i} \cap V_{j}=\varnothing$ for $i \neq j$,

then there is a homeomorphism $f$ of $E^{3}$ onto itself such that $f(K) \subset U$ and $f$ is the identity on $E^{3}-\left(\bigcup_{i=1}^{n} V_{i}\right)$.

The proof of Lemma 2 is omitted as it is obtained directly with an induction argument.

THEOREM 2. If $A$ and $B$ are flexible dendrites in $E^{3}$ such that $A \cap B=\{p\}$, then $A \cup B$ is a flexible dendrite.

Proof. It is clear that $A \cup B$ is a dendrite. To show that $A \cup B$ is flexible let $K_{1}$ and $K_{2}$ be subcontinua of $A \cup B$ such that $A \cup B=$ $K_{1} \cup K_{2}$ and let $U_{1}$ and $U_{2}$ be open sets in $E^{3}$ such that $K_{i} \subset U_{i}$ $(i=1,2)$. We consider separately the cases when $p \notin K_{2}$ and when $p \in K_{2}$ •

Case 1. If $p \notin K_{2}$, then $K_{2} \subset A$ or $K_{2} \subset B$. Let us say that $K_{2} \subset A$. Hence $B \subset K_{1}$. Using the flexibility of $A$ for the subcontinua $K_{1} \cap A$ and $K_{2} \cap A$ and for the open sets $U_{1}$ and $U_{2}$, let $g$ be a homeomorphism of $E^{3}$ onto itself such that $g(A) \subset U_{2}$ and $g$ is the identity on $E^{3}-U_{1}$. Here we used the fact that $K_{i} \cup A(i=1,2)$ is a dendrite and thus unicoherent to say that $K_{i} \cap A$ is a subcontinuum of $A$. Let $N$ be a subcontinuum of $B$ such that $N$ is a neighborhood of $p$ in $B$ and $N \subset g^{-1}\left(U_{2}\right)$. Let $C_{1}, \cdots, C_{n}$ be the components of $\mathrm{Cl}(B-N)$, 
and let $V_{1}, \cdots, V_{n}$ be disjoint open sets in $U_{1}-A$ such that $C_{i} \subset V_{i}$ $(1 \leqq i \leqq n)$. By Lemma 2 , for the flexible dendrite $B$, for the subcontinua $N, C_{1}, C_{2}, \cdots, C_{n}$, and for the open sets $g^{-1}\left(U_{2}\right), V_{1}, V_{2}, \cdots, V_{n}$, there is a homeomorphism $h$ of $E^{3}$ onto itself such that $h(B) \subset g^{-1}\left(U_{2}\right)$ and $h$ is the identity on $E^{3}-\left(\bigcup_{i=1}^{n} V_{i}\right)$. If $f$ equals $g \circ h$, then $f(A \cup B) \subset$ $U_{2}$ and $f$ is the identity on $E^{3}-U_{1}$.

Case 2. If $p \in K_{2}$, then let $N$ be a subcontinuum of $A \cup B$ such that $N$ is a neighborhood of $K_{2}$ in $A \cup B$ and $N \subset U_{2}$. Let $C_{1}, \cdots, C_{n}$ be the components of $\mathrm{Cl}((A \cup B)-N)$. We assume that the set $\left\{C_{i}\right\}_{i=1}^{n}$ is so numbered that for each $i(1 \leqq i \leqq m) C_{i} \subset A-B$ and for each $i(m+1 \leqq i \leqq n) C_{i} \subset B-A$. Let $V_{1}, \cdots, V_{m}$ be disjoint open sets in $U_{1}-B$ such that $C_{i} \subset V_{i}(1 \leqq i \leqq m)$. By Lemma 2 for the flexible dendrite $A$, for the subcontinua $N \cap A, C_{1}, C_{2}, \cdots, C_{m}$, and for the open sets $U_{2}, V_{1}, V_{2}, \cdots, V_{m}$, there is a homeomorphism $g$ of $E^{3}$ onto itself such that $g(A) \subset U_{2}$ and $g$ is the identity on $E^{3}-\left(\bigcup_{i=1}^{m} V_{i}\right)$. Let $V_{m+1}, \cdots, V_{n}$ be disjoint open sets in $U_{1}-g(A)$ such that $C_{i} \subset V_{i}$ $(m+1 \leqq i \leqq n)$. By Lemma 2 for the flexible $B$, for the subcontinua $N \cap B, C_{m+1}, C_{m+2}, \cdots, C_{n}$, and for the open sets $U_{2}, V_{m+1}, V_{m+2}, \cdots, V_{n}$, there is a homeomorphism $h$ of $E^{3}$ onto itself such that $h(B) \subset U_{2}$ and $h$ is the identity on $E^{3}-\left(\bigcup_{i=m+1}^{n} V_{i}\right)$. If $f$ equals $h \circ g$, then $f(A \cup B) \subset U_{2}$ and $f$ is the identity on $E^{3}-U_{1}$.

As a result of Cases 1 and 2, we conclude that $A \cup B$ is flexible.

REMARK. The union of two tame arcs in $E^{3}$ whose intersection is a point need not be a tame dendrite [1, Example 1.4]. Hence there are flexible dendrites in $E^{3}$ which are not tame.

Lemma 3. If $N$ is a tree, then the vertexes of $N$ can be numbered $v_{1}, \cdots, v_{n}$ such that for each $i(1 \leqq i \leqq n-1)$, there is a unique integer $s(i)$ satisfying $i<s(i) \leqq n$ and there is a 1-simplex between $v_{i}$ and $v_{s(i)}$.

Proof. The proof is by induction on the number of vertexes of $N$. Any numbering works if $N$ has two vertexes. Assume the lemma is true if $N$ has $n-1(n \geqq 3)$ vertexes, and consider the case when $N$ has $n$ vertexes. Let $w$ be a vertex of $N$ which is the face of exactly one 1-simplex $s$ in $N$. We form a new tree $N^{\prime}$ by removing $w$ and the interior of $s$ from $N$. By the induction hypothesis we can number the vertexes $u_{1}, \cdots, u_{n-1}$ of $N^{\prime}$ such that for each $i(1 \leqq i \leqq$ $n-2)$, there is a unique integer $s(i)$ satisfying $i<s(i) \leqq n-1$ and there is a 1-simplex between $u_{i}$ and $u_{s(i)}$. Now in $N$ let $v_{1}=w$ and let $v_{i}=u_{i-1}(2 \leqq i \leqq n)$. This numbering satisfies the condition. 
Lemma 4. Let $A$ be a dendrite and let $\varepsilon$ be a positive real number. Then $A$ is the finite union of continua $A_{1}, \cdots, A_{n}$ of diameter less then $\varepsilon$ such that for each $i(1 \leqq i \leqq n-1)$, there is a unique integer $s(i)$ satisfying $i<s(i) \leqq n$ and $A_{i} \cap A_{s(i)} \neq \varnothing$.

Proof. The dendrite $A$ can be written as the finite union of continua $A_{1}, \cdots, A_{n}$ of diameter less than $\varepsilon$ such that each pair intersects in at most a point and each triplet has empty intersection [3, p. 302]. It follows that the nerve $N$ of $\left\{A_{i}\right\}_{i=1}^{n}$ is a tree. Using Lemma 3 we see that the set $\left\{A_{i}\right\}_{i=1}^{n}$ can be renumbered such that for each $i(1 \leqq i \leqq n-1)$, there is a unique integer $s(i)$ satisfying $i<s(i) \leqq n$ and $A_{i} \cap A_{s(i)} \neq \varnothing$.

THeorem 3. If $G$ is an upper semicontinuous decomposition of $E^{3}$ whose only nondegenerate elements are countably many flexible dendrites, then $E^{3} / G$ is homeomorphic to $E^{3}$.

Proof. Using the technique of Bing as in [2, Theorem 3], it suffices to show that if $G$ is an upper semicontinuous decomposition of $E^{3}, \varepsilon$ is a positive real number, $A$ is an element of $G$ which is a flexible dendrite, and $U$ is an open set containing $A$, then there is a homeomorphism $f$ of $E^{3}$ onto itself such that $f$ is the identity on $E^{3}-U$, diam $f(A)<\varepsilon$, and for each element $g$ of $G$, either diam $f(g)<\varepsilon$ or $f(g) \subset N(g, \varepsilon)$ where $N(g, \varepsilon)=\left\{x \in E^{3}\right.$ : dist $\left.(x, g)<\varepsilon\right\}$.

By Lemma 4 the dendrite $A$ is the finite union of continua $A(1)_{1}, \cdots, A(1)_{n}$ of diameter less than $\varepsilon$ such that for each $i(1 \leqq i \leqq$ $n-1)$, there is a unique integer $s(i)$ satisfying $i<s(i) \leqq n$ and $A(1)_{i} \cap A(1)_{s(i)} \neq \varnothing$. We may assume that $n>1$, otherwise $f$ equals to the identity on $E^{3}$ would be the required homeomorphism. For each $i(1 \leqq i \leqq n)$ let $U(1)_{i}$ be an open set in $E^{3}$ such that $A(1)_{i} \subset$ $U(1)_{i} \subset U$, diam $U(1)_{i}<\varepsilon$, and $\mathrm{Cl} U(1)_{i} \cap \mathrm{Cl} U(1)_{j}=\varnothing$ if and only if $A(1)_{i} \cap A(1)_{j}=\varnothing$. Since $A$ is flexible, for the subcontinua $A(1)_{1}$ and $\bigcup_{i=2}^{n} A(1)_{i}$ and for the open sets $U(1)_{1}$ and $\bigcup_{i=2}^{n} U(1)_{i}$, there is a homeomorphism $f_{1}$ of $E^{3}$ onto itself such that $f_{1}(A) \subset \bigcup_{i=2}^{n} U(1)_{i}$ and $f_{i}$ is the identity on $E^{3}-U(1)_{1}$. Once given $\left\{A(j)_{i}\right\}_{i=j}^{n},\left\{U(j)_{i}\right\}_{i=j}^{n}$, and $f_{j}$ for fixed $j(1 \leqq j \leqq n-2)$, define for each $i(j+1 \leqq i \leqq n)$

$$
A(j+1)_{i}= \begin{cases}f_{j}\left(A(j)_{i}\right)=A(j)_{i} & \text { if } i \neq s(j) \\ f_{j}\left(A(j)_{j} \cup A(j)_{s(j)}\right) & \text { if } i=s(j) .\end{cases}
$$

Also for each $i(j+1 \leqq i \leqq n)$, let $U(j+1)_{i}$ be an open set in $E^{3}$ such that:

(1) $A(j+1)_{i} \subset U(j+1)_{i} \subset U(j)_{i}$, and

(2) $\bigcup_{g \in G}\left\{g_{j}: g_{j}\right.$ meets $\left.U(j+1)_{i}\right\} \subset \bigcup_{k=j+1}^{n} U(j)_{k}$, where $g_{j}$ denotes $f_{j} \circ \cdots \circ f_{1}(g)$. 
Condition (2) can be satisfied since $f_{j} \circ \cdots \circ f_{1}(A)$ which equals $\bigcup_{k=j+1}^{n} A(j+1)_{k}$ is an element of the upper semicontinuous decomposition $G_{j}=\left\{f_{j} \circ \cdots \circ f_{1}(g): g \in G\right\}$ and a subset of the open set $\bigcup_{k=j+1}^{n} U(j)_{k}$.

Using the flexibility of $f_{j} \circ \cdots \circ f_{1}(A)$ for the subcontinua $A(j+1)_{j+1}$ and $\bigcup_{i=j+2}^{n} A(j+1)_{i}$ and the open sets $U(j+1)_{j+1}$ and $\bigcup_{i=j+2}^{n} U(j+1)_{i}$ obtain a homeomorphism $f_{j+1}$ of $E^{3}$ onto itself such that

$$
f_{j+1}\left(f_{j} \circ \cdots \circ f_{1}(A)\right) \subset \bigcup_{i=j+2}^{n} U(j+1)_{i}
$$

and $f_{j+1}$ is the identity on $E^{3}-U(j+1)_{j+1}$. Let $f$ equal $f_{n-1} \circ \cdots \circ f_{1}$. We wish to show that $f$ is the required homeomorphism.

It is clear that $f$ is the identity on $E^{3}-U$ and diam $f(A)<\varepsilon$. Hence we show that if $g \in G$, then $\operatorname{diam} f(g)<\varepsilon$ or $f(g) \subset N(g, \varepsilon)$. Since $f_{1}$ is the identity on $E^{3}-U(1)_{1}, f_{1}$ moves no point of $E^{3}$ more than diam $U(1)_{1}<\varepsilon$. Hence $f_{1}(g) \subset N(g, \varepsilon)$. Suppose now we have proven for fixed $k(2 \leqq k \leqq n-1)$ that diam $g_{k-1}<\varepsilon$ or $g_{k-1} \subset N(g, \varepsilon)$ where $g_{k-1}$ denotes $f_{k-1} \circ \cdots \circ f_{1}(g)$. We show that diam $g_{k}<\varepsilon$ or $g_{k} \subset N(g, \varepsilon)$. If $g_{k-1}$ does not meet $U(k)_{k}$, then $g_{k}$ equals $g_{k-1}$. Thus diam $g_{k}<\varepsilon$ or $g_{k} \subset N(g, \varepsilon)$. If $g_{k-1}$ meets $U(k)_{k}$, then $g_{k-1} \subset \bigcup_{j=k} U(k-1)_{j}$ by condition (2). We consider two cases.

Case 1. If $g_{k-1} \subset U(k-1)_{k}$, then since $f_{k}$ is the identity on $E^{3}-$ $U(k)_{k}$ and $U(k)_{k} \subset U(k-1)_{k}$, we have $g_{k} \subset U(k-1)_{k}$. Thus diam $g_{k}<\varepsilon$.

Case 2. If $g_{k-1}$ meets $\left(\bigcup_{j=k}^{n} U(k-1)_{j}\right)-U(k-1)_{k}$, then $g_{k-1}$ meets the boundary $B$ of $U(k-1)_{k}$ as a subset of $\bigcup_{j=k}^{n} U(k-1)_{j}$. Let $y \in B \cap g_{k-1}$. We wish to show that $y \in g$. For each $i(1 \leqq i \leqq k-1)$, since there is only one integer $s(i)$ such that $i<s(i) \leqq n$ and $A(i)_{i} \cap$ $A(i)_{s(i)} \neq \varnothing$, either $\mathrm{Cl} U(i)_{i} \cap \mathrm{Cl} U(k-1)_{k}=\varnothing$ or

$$
\mathrm{Cl} U(i)_{i} \cap\left(\bigcup_{j=k+1}^{n} \mathrm{Cl} U(k-1)_{j}\right)=\varnothing \text {. }
$$

Hence for each $i(1 \leqq i \leqq k-1), \mathrm{Cl} U(i)_{i} \cap B=\varnothing$, and thus $f_{i}$ is the identity on $B$. Hence $y \in g$. We now show that $g_{k} \subset N(g, \varepsilon)$ by proving if $x \in g_{k-1}$, then $\operatorname{dist}\left(f_{k}(x), g\right)<\varepsilon$. If $x \in U(k-1)_{k}$, then $f_{k}(x) \in$ $U(k-1)_{k}$. Hence

$$
\operatorname{dist}\left(f_{k}(x), g\right) \leqq \operatorname{dist}\left(f_{k}(x), y\right) \leqq \operatorname{diam}\left(\mathrm{Cl} U(k-1)_{k}\right)<\varepsilon .
$$

If $x \notin U(k-1)_{k}$, then $f_{k}(x)=x$, and we must consider the cases when diam $g_{k-1}<\varepsilon$ and when $g_{k-1} \subset N(g, \varepsilon)$ separately. If diam $g_{k-1}<\varepsilon$, then

$$
\operatorname{dist}\left(f_{k}(x), g\right) \leqq \operatorname{dist}(x, y) \leqq \operatorname{diam} g_{k-1}<\varepsilon .
$$

If $g_{k-1} \subset N(g, \varepsilon)$, then 


$$
\operatorname{dist}\left(f_{k}(x), g\right)=\operatorname{dist}(x, g)<\varepsilon .
$$

Hence we have shown that $g_{k} \subset N(g, \varepsilon)$.

As a result of Cases 1 and 2, we can conclude by induction that if $g \in G$, then $\operatorname{diam} f(g)<\varepsilon$ or $f(g) \subset N(g, \varepsilon)$. Thus $f$ is the required homeomorphism.

Definition. A continuum $K$ in $E^{3}$ is cellular if there is a sequence of 3 -cells $\left\{C_{i}\right\}_{i=1}^{\infty}$ in $E^{3}$ such that $K=\bigcap_{i=1}^{\infty} C_{i}$ and $C_{i+1} \subset \operatorname{Int} C_{i}$ for $i=1,2, \cdots$.

Corollary. If $K$ is a flexible dendrite in $E^{3}$, then $K$ is cellular.

Proof. Let $G$ be an upper semicontinuous decomposition of $E^{3}$ into continua with only countably many nondegenerate elements. By Theorem 2 of [4] if $E^{3} / G$ is homeomorphic to $E^{3}$, then each element of $G$ is cellular.

REMARK. For an example of a cellular dendrite which is not, flexible consider the cellular are $A$ of Example 1.2 in [1]. This are has only one wild point, an endpoint. To see that this are is not flexible, consider another arc $B$ in $E^{3}-A$ such that $A$ and $B$ are equivalently embedded in $E^{3}$ under a space homeomorphism of $E^{3}$. Let $J$ be a tame arc in $E^{3}$ which joins the locally tame endpoint of $A$ to the locally tame endpoint of $B$ to form an arc $K=A \cup J \cup B$. If $A$ is flexible, then by Theorem 2 the arc $K$ is flexible. Hence $K$ is cellular. However, a cellular arc in $E^{3}$ cannot have isolated wild points for its endpoints [5, Theorem 10]. Thus $A$ is not fiexible.

\section{REFERENCES}

1. E. Artin and R. H. Fox, Some wild cells and spheres in three-dimensional space, Ann. of Math., (2) $\mathbf{4 9}$ (1948), 979-990.

2. R. H. Bing, Upper semicontinuous decompositions of $E^{3}$, Ann. of Math., (2) 65 (1957), 363-374.

3. K. Kurotowski, Topology, vol. 2, English translation, Academic Press, N. I.. 1963.

4. K. W. Kwun, Upper semi-continuous decompositions of the n-sphere, Proc. Amer. Math. Soc., 13 (1962), 284-290.

5. C. D. Sikkema, $A$ a'uality between ceriain spheres and arcs in $S^{3}$, Trans. Amer. Math. Soc., 122 (1966), 399-415.

Received July 14, 1972 and in revised form December 11, 1972. This work is contained in the author's doctoral dissertation submitted to Indiana University, and was supported by NSF Traineeship GZ2000. The author wishes to thank his advisor, Professor Arlo Schurle, for his assistance throughout the work.

\section{INDIANA UNIVERSITY}

Current Address: University of North Carolina at Charlotte. 



\section{PACIFIC JOURNAL OF MATHEMATICS}

\section{EDITORS}

RICHARD ARENS (Managing Editor)

University of California

Los Angeles, California 90024

\author{
R. A. Beaumont \\ University of Washington \\ Seattle, Washington 98105
}

J. Dugundu*

Department of Mathematics

University of Southern California

Los Angeles, California 90007

D. Gilbarg and J. Milgram

Stanford University

Stanford, California 94305

\section{ASSOCIATE EDITORS}
E. F. BECKENBACH
B. H. NeumanN
F. WOLF
K. YosHIDA

\section{SUPPORTING INSTITUTIONS}

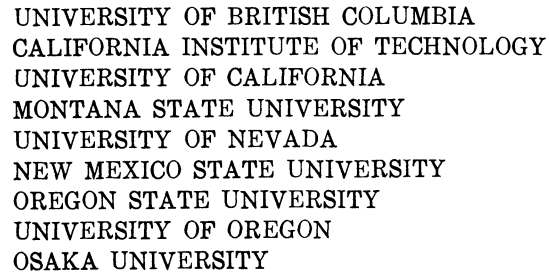

UNIVERSITY OF BRITISH COLUMBIA CALIFORNIA INSTITUTE OF TECHNOLOGY

UNIVERSITY OF CALIFORNIA

MONTANA STATE UNIVERSITY

UNIVERSITY OF NEVADA

NEW MEXICO STATE UNIVERSITY

OREGON STATE UNIVERSITY

UNIVERSITY OF OREGON

OSAKA UNIVERSITY

\author{
UNIVERSITY OF SOUTHERN CALIFORNIA \\ STANFORD UNIVERSITY \\ UNIVERSITY OF TOKYO \\ UNIVERSITY OF UTAH \\ WASHINGTON STATE UNIVERSITY \\ UNIVERSITY OF WASHINGTON \\ * * * \\ AMERICAN MATHEMATICAL SOCIETY \\ NAVAL WEAPONS CENTER
}

The Supporting Institutions listed above contribute to the cost of publication of this Journal, but they are not owners or publishers and have no responsibility for its content or policies.

Mathematical papers intended for publication in the Pacific Journal of Mathematics should be in typed form or offset-reproduced, (not dittoed), double spaced with large margins. Underline Greek letters in red, German in green, and script in blue. The first paragraph or two must be capable of being used separately as a synopsis of the entire paper. Items of the bibliography should not be cited there unless absolutely necessary, in which case they must be identified by author and Journal, rather than by item number. Manuscripts, in duplicate if possible, may be sent to any one of the four editors. Please classify according to the scheme of Math. Rev. Index to Vol. 39. All other communications to the editors should be addressed to the managing editor, or Elaine Barth, University of California, Los Angeles, California, 90024.

50 reprints are provided free for each article; additional copies may be obtained at cost in multiples of 50 .

The Pacific Journal of Mathematics is issued monthly as of January 1966. Regular subscription rate: $\$ 48.00$ a year (6 Vols., 12 issues). Special rate: $\$ 24.00$ a year to individual members of supporting institutions.

Subscriptions, orders for back numbers, and changes of address should be sent to Pacific Journal of Mathematics, 103 Highland Boulevard, Berkeley, California, 94708.

PUBLISHED BY PACIFIC JOURNAL OF MATHEMATICS, A NON-PROFIT CORPORATION

Printed at Kokusai Bunken Insatsusha (International Academic Printing Co., Ltd.), 270, 3-chome Totsuka-cho. Shinjuku-ku, Tokyo 160, Japan.

* C. R. DePrima California Institute of Technology, Pasadena, CA 91109, will replace J. Dugundji until August 1974.

Copyright (C) 1973 by

Pacific Journal of Mathematics

All Rights Reserved 


\section{Pacific Journal of Mathematics}

Vol. 48, No. $2 \quad$ April, 1973

Mir Maswood Ali, Content of the frustum of a simplex................

Mieczyslaw Altman, Contractors, approximate identities and factorization

in Banach algebras ................................ 323

Charles Francis Amelin, A numerical range for two linear operators ...... 335

John Robert Baxter and Rafael Van Severen Chacon, Nonlinear functionals

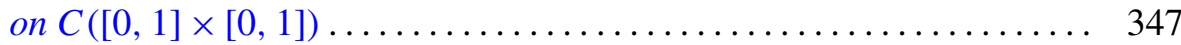

Stephen Dale Bronn, Cotorsion theories....................... 355

Peter A. Fowler, Capacity theory in Banach spaces............... 365

Jerome A. Goldstein, Groups of isometries on Orlicz spaces ........... 387

Kenneth R. Goodearl, Idealizers and nonsingular rings . ............ 395

Robert L. Griess, Jr., Automorphisms of extra special groups and

nonvanishing degree 2 cohomology ..................... 403

Paul M. Krajkiewicz, The Picard theorem for multianalytic functions . . . . 423

Peter A. McCoy, Value distribution of linear combinations of axisymmetric harmonic polynomials and their derivatives ...................

A. P. Morse and Donald Chesley Pfaff, Separative relations for

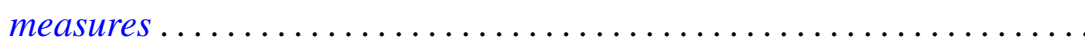

Albert David Polimeni, Groups in which $\operatorname{Aut}(G)$ is transitive on the

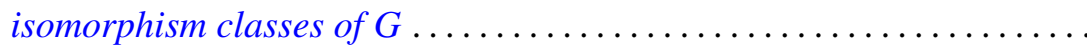

Aribindi Satyanarayan Rao, Matrix summability of a class of derived

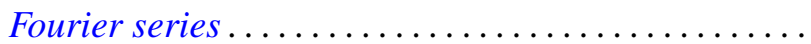

Thomas Jay Sanders, Shape groups and products

Ruth Silverman, Decomposition of plane convex sets. II. Sets associated

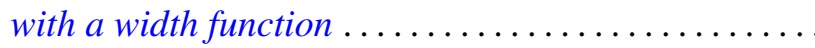

Richard Snay, Decompositions of $E^{3}$ into points and countably many flexible dendrites.............................

John Griggs Thompson, Nonsolvable finite groups all of whose local subgroups are solvable, IV ...

Robert E. Waterman, Invariant subspaces, similarity and isometric equivalence of certain commuting operators in $L_{p} \ldots$

James Chin-Sze Wong, An ergodic property of locally compact amenable

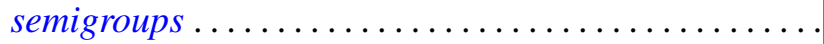

Julius Martin Zelmanowitz, Orders in simple Artinian rings are strongly equivalent to matrix rings ....................... 